\title{
On the solution of multi-dimensional nonlinear integral equation with modified newton method
}

\begin{abstract}
In this note, multi-dimensional nonlinear integral equations (multi-dimensional NIEs) in $\mathrm{R} \mathrm{n}$ is considered. Implementing modified Newton method (Modified NM) reduces multidimensional NIEs into multi-dimensional linear integral equations (multi-dimensional LIEs), which can be solved by discritization method. Quadrature method together with collocation are used to find values of unknown functions. Existence and uniqueness solution of the problems are shown. The rate of convergence of the proposed method is proved using the principle of majorant function. Finally, numerical examples are provided and it reveals that proposed method is both accurate and effective as well as comparisons with other methods are also presented.
\end{abstract}

Keyword: Nonlinear multi-dimensional integral equations; Quadrature formula; The majorant function; The modified newton method 